\title{
Avaliação do desenvolvimento motor de crianças com dificuldades de aprendizagem
}

\author{
Evaluation of motor development in children with learning \\ disabilities
}

\section{Universidade Estadual de Londrina. Depar- tamento de Educação Física. Londrina, PR. Brasil. \\ 2. Universidade Estadual de Londrina. Curso de Mestrado em Educação Física. Grupo de Estudo e Pesquisa em Desenvol- vimento e Aprendizagem Motora. Londrina, PR. Brasil.}

Recebido em 03/09/08 Revisado em 12/03/09 Aprovado em 22/06/09
Resumo - O objetivo do estudo foi investigar se crianças com dificuldades de aprendizagem apresentam algum comprometimento motor no desenvolvimento dos componentes da motricidade. Participaram da pesquisa, trinta crianças, 21 meninos e 9 meninas, de 8 a 10 anos de idade, com dificuldades de aprendizagem escolar. As crianças foram avaliadas por meio da Escala de Desenvolvimento Motor para avaliação do desenvolvimento nos componentes de motricidade fina, motricidade global, equilíbrio, esquema corporal, organização espacial e organização temporal. Verificou-se maior atraso no desenvolvimento do esquema corporal para os três grupos etários, e maior idade motora no equilíbrio e motricidade global. Não foram identificadas diferenças significativas na idade motora geral entre os grupos etários. Concluiu-se que essas crianças apresentam atraso motor no desenvolvimento da maioria dos componentes avaliados, em especial, as crianças mais velhas. Sugere-se a inclusão de tarefas que auxilie no desenvolvimento dos componentes motores, além das tarefas escolares.

Palavras-chave: Desenvolvimento infantil; Aprendizagem; Avaliação; Movimento, Destreza motora.

Abstract - The aim of this study was to determine whether children with learning disabilities present any impairment in the components of motor development. Thirty children (21 boys and 9 girls), aged 8 to 10 years, with learning difficulties in school were studied. The Motor Development Scale was used to evaluate the development of the children in terms of fine motor control, gross motor control, balance, body scheme, spatial organization, and temporal organization. A deficit in the development of the body scheme component was observed for all three age groups, as well as a delayed motor development in terms of balance and gross motor control. No significant differences in general motor age were observed between (age) groups. In conclusion, the children studied, especially older ones, presented motor deficits in most of the components evaluated. The inclusion of tasks that assist in the development of motor components, in addition to regular school tasks, is recommended to improve the process of learning in these children.

Key words: Child development; Learning; Evaluation; Movement; Motor skills 


\section{INTRODUÇÃO}

A experiência motora propicia o amplo desenvolvimento dos diferentes componentes da motricidade, tais como a coordenação, o equilíbrio e o esquema corporal. Esse desenvolvimento é fundamental, particularmente, na infância, para o desenvolvimento das diversas habilidades motoras básicas como andar, correr, saltar, galopar, arremessar e rebater. No entanto, embora o desenvolvimento motor infantil não ocorra de forma linear ${ }^{1,2}$, é fundamental que se ofereça à criança um ambiente diversificado, de situações novas e que propicie meios diversos de resolução de problemas, uma vez que o movimento se apresenta e se aprimora por meio dessa interação, das mudanças individuais com o ambiente e a tarefa motora ${ }^{1}$.

O problema é que os alunos são diferentes, exigindo do professor diferentes estratégias de ensino de forma a tornar a prática eficiente para todos ${ }^{3}$. Ressalta-se a importância do desenvolvimento das habilidades motoras e dos aspectos referentes à sua aplicação nas tarefas escolares, visto que deficiências em determinados campos do desenvolvimento podem gerar atrasos e influenciar no avanço em tarefas de outras áreas relacionadas à aprendizagem em geral. A Educação Física adquire, assim, um papel importante na medida em que pode estruturar o ambiente adequado para a criança refletir sobre suas ações e experiências práticas, funcionando como uma grande auxiliar e promotora do desenvolvimento humano e, em especial, do desenvolvimento motor ${ }^{4}$. Assim, para que um programa de atividades atinja os objetivos esperados em relação aos componentes motores das crianças, há a necessidade de investigação sobre o nível de desenvolvimento motor das mesmas.

Estudos sobre o desenvolvimento da motricidade infantil, em geral, são realizados com o objetivo de conhecer o perfil motor das crianças e de poder estabelecer instrumentos de confiança para avaliar e analisar o desenvolvimento de alunos em diferentes etapas do seu desenvolvimento 5 . Com esta finalidade, a avaliação motora torna-se um importante instrumento que favorece o conhecimento de dados relacionados ao desenvolvimento motor da criança e sugere estratégias de integração de atividades relacionadas às necessidades específicas de cada uma. Os testes de avaliação do desenvolvimento motor utilizam critérios de seleção variados, como a idade da criança e a área a ser avaliada (força muscular, motricidade fina, motricidade ampla, fala, ou avaliação abrangente das capacidades funcionais) e agem facilitando o planejamento e formas de intervenção ${ }^{6}$.

Uma situação que gera preocupação e tem atingido grande parte de escolares das séries iniciais do ensino básico, é a incidência de crianças com dificuldades de aprendizagem escolar. Preocupações importantes são manifestadas por pais e professores referentes ao insucesso escolar e às dificuldades de aprendizagem das crianças que, muitas vezes, se refletem em frustrações e problemas de maior proporção. Essas dificuldades podem não estar atreladas a nenhum tipo de comprometimento neurológico ou estrutural ${ }^{6}$ como doenças congênitas, atrasos mentais ou deficiência física, entretanto, definem o desempenho da criança nas diversas atividades escolares.

Alguns estudos destacam mudanças no comportamento afetivo e tendência ao desenvolvimento de uma autopercepção de competência negativa, enquanto outros demonstram a implicação deste quadro sobre o desenvolvimento de aspectos cognitivos e da motricidade ${ }^{7-10}$. Na maioria das vezes, os problemas de aprendizagem residem sobre as áreas de percepção, atenção, memória, associação e fixação de informações, os quais podem perdurar na vida jovem e adulta ${ }^{11}$. Além disso, crianças que apresentam dificuldades de aprendizagem em leitura e escrita, na sua maioria, possuem a mesma forma de relacionar-se com as outras áreas trabalhadas no contexto escolar ${ }^{12}$.

Assim, há de se considerar que na Educação Física Escolar, o nível de desenvolvimento motor e os componentes da motricidade das crianças são fatores preponderantes para investigação. Associado a esses temas, os problemas de dificuldades de aprendizagem também se estabelecem como aspectos importantes, uma vez que há estudos que destacam o atraso no desenvolvimento motor e mudanças no controle do movimento, decorrentes desses problemas.

Nesse sentido, o objetivo desse trabalho foi investigar se crianças com dificuldades de aprendizagem apresentam algum comprometimento motor no desenvolvimento dos componentes da motricidade.

\section{PROCEDIMENTOS METODOLÓGICOS}

A amostra compreendeu 30 crianças da rede pública de ensino, 21 meninos e 9 meninas, de 8 a 10 anos de idade, com dificuldades de aprendizagem escolar relatadas pela professora de sala e diagnosticadas a partir do nível de desenvolvimento da leitura, escrita e avaliação do seu desempenho escolar por meio de análise de produções escolares do aluno. A avaliação das dificuldades de aprendizagem das crianças indicadas pelas professoras é comumente realizada 
por estudantes de psicopedagogia e, quando confirmadas, as crianças são distribuídas nas sessões de atendimento especial de um programa psicopedagógico, realizado em horário extra-turno, na escola. Assim, após a consulta das suas fichas cadastrais, nas quais se constatou que elas não tinham diagnóstico sobre qualquer atraso cognitivo ou deficiência mental, as crianças foram convidadas a participar das tarefas de avaliação. Os pais ou responsáveis assinaram um termo de consentimento livre e esclarecido para a participação das crianças no estudo, o qual foi aprovado pelo Comitê de Ética e Pesquisa com Seres Humanos (UEL - n 242/07).

As crianças foram distribuídas em grupos de acordo com a idade cronológica, sendo o grupo de 8 anos (96-107 meses, média 100,49 $\pm 3,52$ meses) composto por 6 meninos e 3 meninas, o grupo de 9 anos (108-119 meses, média de 114,82 $\pm 3,74$ meses) 5 meninos e 3 meninas e o grupo de 10 anos (120-131 meses, média $126,48 \pm 4,25$ meses) composto por 10 meninos e 3 meninas.

Para a análise do desenvolvimento motor, foram avaliadas habilidades referentes ao desenvolvimento da motricidade fina, motricidade global, equilíbrio, esquema corporal, organização espacial e organização temporal, seguindo-se a ordem proposta no Manual de Avaliação Motora ${ }^{5}$. O teste foi iniciado pela idade cronológica da criança e quando o êxito era obtido, avançava-se para as tarefas relativas às idades seguintes até que um erro fosse detectado. Quando a criança não obtivesse êxito na primeira tentativa, recorria-se às tarefas pertinentes às idades anteriores até a obtenção de sucesso pela criança. As avaliações foram realizadas, individualmente, por quatro avaliadoras treinadas, anteriormente, e os dados das crianças foram anotados em uma ficha e, posteriormente, analisados para cálculo dos resultados.

As avaliações foram realizadas na própria escola, em dias agendados anteriormente, com a orientação educacional. Foi recomendado às crianças o uso de roupas que não dificultassem os movimentos durante os testes. Caso a criança não estivesse usando tênis, solicitou-se tirar o calçado para a execução das provas.

\section{Análise dos dados}

Os dados foram tabulados por Idade Cronológica (IC), em meses e pelas respectivas idades motoras (IM), em cada tarefa, cujo resultado é obtido com base nas tabelas normativas. ${ }^{5}$ A idade motora geral (IMG) foi obtida a partir da razão entre a soma das idades motoras e o número de tarefas realizadas.
A estatística descritiva foi utilizada adotandose os valores medianos, mínimos e máximos, após verificação de dados não normais por meio do teste de Shapiro-Wilk. O teste de Friedman foi utilizado para verificar as diferenças entre a IC e Idade motora (IM) obtida em cada teste, nos grupos de 96, 108 e 120 meses, e o teste de Wilcoxon foi utilizado como post hoc para localizar tais diferenças. Para verificar diferenças da IMG entre os grupos, utilizou-se o teste de Kruskall-Wallis. O nível de significância adotado foi de $\mathrm{p}<0,05$.

\section{RESULTADOS}

Os resultados apresentados na Figura 1 demonstram a análise descritiva da amostra com idade cronológica (IC) e a distribuição das crianças com base na mediana da idade motora (IM) em cada teste.

O valor da mediana da IC foi de 118,3 meses, com mínimo de 96,3 e máximo de 131,8 meses, enquanto a idade motora geral (IMG) das crianças apresentou valor mediano de 100,5 meses, mínimo de 84,0 e máximo de 123,0 meses. Pode-se verificar que a IM foi inferior a IC em todos os testes avaliados.

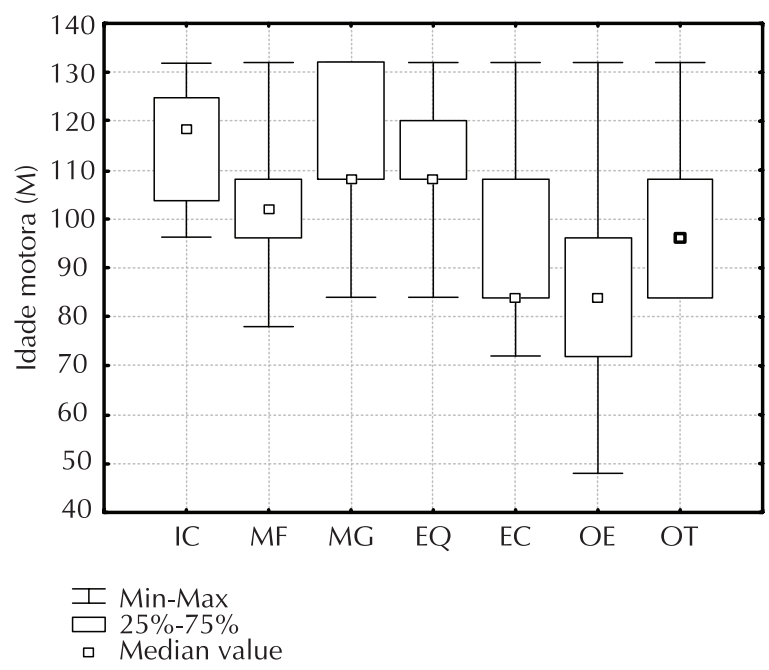

Figura 1. Mediana, mínimo e máximo da idade cronológica (IC) e da idade motora (IM) nos testes de motricidade fina (MF), motricidade global (MG), equilíbrio (EQ), esquema corporal (EC), organização espacial (OE) e organização temporal (OT) de toda a amostra.

No teste de motricidade global, a maior parte da amostra encontrou-se próxima aos valores máximos obtidos nesse teste. Entretanto, o inverso pode ser observado no teste de organização temporal, em que a maioria se aproxima dos valores mínimos encontrados para a IM das crianças. Particularmente sobre esses resultados, sugere-se que grande parte das crianças avaliadas encontra-se com IM mais avançada no teste de motricidade global, em relação ao teste 
de organização temporal. Dessa forma, a amostra foi distribuída em grupos etários, 96-107, 108-119 e 120-131 meses, a fim de analisar as diferenças entre a IC e a IM ,em cada teste, nos três grupos.

No grupo de crianças de 96 meses de idade (mediana 99,2 meses), verificou-se diferença significativa entre a IC e o desenvolvimento motor nos testes de esquema corporal $(Z=2,66 ; p=0,00)$, organização espacial $(Z=2,66 ; p=0,00)$ e organização temporal $(Z=2,19$; $\mathrm{p}=0,02$ ), sendo que a menor IM encontrada foi no teste de organização espacial (72,0 meses). Todavia, a IMG não foi estatisticamente diferente da IC das crianças.

Similarmente, o grupo de crianças com 108 meses (mediana 115,1 meses) demonstrou a menor IM no teste de organização espacial (90,0 meses). Houve diferença significativa entre a IC e o desenvolvimento motor nos testes de motricidade fina $(Z=2,52 ; p=0,01)$, esquema corporal $(Z=2,52$; $p=0,01)$, organização espacial $(Z=2,38 ; p=0,01)$ e na IMG $(Z=2,52 ; p=0,01)$. A mediana da IMG desse grupo foi inferior à mediana de $\mathrm{IC}$, com diferença de 13,6 meses (Tabela 2).

Os resultados do grupo de crianças de 120 meses de idade (mediana 127,7 meses) demonstraram valores de IM, significativamente, inferiores à IC, em todos os componentes da motricidade $(\mathrm{p}<0,05)$, com exceção do

Tabela 1. Valores de média $(X)$, mediana, mínimo $(M I N)$ e máximo $(M A X)$ das idades motoras obtidas pelas crianças com idade cronológica (IC) de 8 anos (96-107 meses).

\begin{tabular}{lccccc}
\hline TESTES & $\mathrm{N}$ & $\mathrm{X}$ & MEDIANA & MIN & MAX \\
\hline IC & 9 & 100,5 & $99,2^{*}$ & 96,3 & 106,0 \\
Motricidade fina & 9 & 102,7 & 102,0 & 78,0 & 132,0 \\
Motricidade global & 9 & 104,0 & 108,0 & 84,0 & 132,0 \\
Equilíbrio & 9 & 104,0 & 108,0 & 84,0 & 132,0 \\
Esquema corporal & 9 & 85,3 & $84,0^{*}$ & 84,0 & 96,0 \\
Organização espacial & 9 & 73,3 & $72,0^{*}$ & 48,0 & 96,0 \\
Organização temporal & 9 & 92,0 & $96,0^{*}$ & 84,0 & 108,0 \\
Idade motora geral (IMG) & 9 & 93,5 & 95,0 & 84,0 & 107,0 \\
\hline
\end{tabular}

$* p<0,05$

Tabela 2. Valores de média (X), mediana, mínimo (MIN) e máximo (MAX) das idades motoras obtidas pelas crianças com idade cronológica (IC) de 9 anos (108-119 meses).

\begin{tabular}{llllll}
\hline TESTES & $\mathrm{N}$ & $\mathrm{X}$ & MEDIANA & MIN & MAX \\
\hline IC & 8 & 114,8 & $115,1^{*}$ & 109,9 & 119,5 \\
Motricidade fina & 8 & 102,7 & 105,0 & 96,0 & 108,0 \\
\hline Motricidade global & 8 & 121,5 & 126,0 & 108,0 & 132,0 \\
Equilíbrio & 8 & 111,0 & 108,0 & 108,0 & 132,0 \\
Esquema corporal & 8 & 93,0 & $96,0^{*}$ & 72,0 & 108,0 \\
Organização espacial & 8 & 87,7 & $90,0^{*}$ & 48,0 & 132,0 \\
Organização temporal & 8 & 99,0 & 96,0 & 84,0 & 120,0 \\
Idade motora geral (IMG) & 8 & 102,5 & $101,5^{*}$ & 94,0 & 110,0 \\
\hline
\end{tabular}

${ }^{*} \mathrm{p}<0,05$

Tabela 3. Valores de média (X), mediana, mínimo (MIN) e máximo (MAX) das idades motoras obtidas pelas crianças com idade cronológica (IC) de 10 anos (120-131 meses).

\begin{tabular}{lccccc}
\hline Testes & $\mathrm{n}$ & $\mathrm{x}$ & Mediana & $\min$ & $\max$ \\
\hline IC & 13 & 126,5 & $127,7^{*}$ & 120,5 & 131,9 \\
Motricidade fina & 13 & 109,0 & $102,0^{*}$ & 84,0 & 132,0 \\
Motricidade global & 13 & 118,1 & 120,0 & 96,0 & 132,0 \\
Equilíbrio & 13 & 115,4 & $108,0^{*}$ & 84,0 & 132,0 \\
Esquema corporal & 13 & 102,7 & $96,0^{*}$ & 72,0 & 132,0 \\
Organização espacial & 13 & 89,5 & $96,0^{*}$ & 60,0 & 108,0 \\
Organização temporal & 13 & 106,1 & $108,0^{*}$ & 84,0 & 132,0 \\
IMG & 13 & 106,7 & $108,0^{*}$ & 88,0 & 123,0 \\
\hline
\end{tabular}

$* p<0,05$ 
teste de motricidade global (Tabela 3). As menores IM encontradas foram nos testes de esquema corporal e organização espacial (96 meses), apresentando diferença de 31,7 meses em relação à mediana da IC das crianças.

De acordo com esses resultados, pôde-se observar que as maiores discrepâncias entre a IC e o desenvolvimento dos componentes da motricidade estão presentes no grupo de crianças mais velhas, no qual se encontrou maiores diferenças. Além disso, esse grupo apresentou maior amplitude entre a mediana de IC e mediana de IMG (127,7 e 108,0 meses, respectivamente).

O teste Kruskall-Wallis não demonstrou diferenças significativas entre a IMG obtida pelos grupos $(\mathrm{p}>0,05)$. Isso reafirma um relativo atraso no desenvolvimento motor das crianças mais velhas.

\section{DISCUSSÃO}

Os resultados demonstraram que os três grupos etários avaliados apresentaram atrasos no desenvolvimento motor, considerando os resultados da IMG. Além disso, quanto maior a idade, maiores os atrasos na maioria dos componentes avaliados. Todavia, sabe-se que o desenvolvimento dos componentes da motricidade está relacionado à interação de diversos fatores, dentre eles, aspectos biológicos ou ambientais ${ }^{4}$, o desenvolvimento de componentes cognitivos, a oportunidade de prática de atividades motoras e fatores alimentares ${ }^{3,13,14}$, além do tempo excessivo gasto somente com jogos televisivos ou até mesmo restrição de atividades como, simetria corporal, equilíbrio postural e outras ${ }^{15}$.

O presente estudo identificou que o resultado dos testes de motricidade global e equilíbrio alcançaram IM mais próxima ao esperado, isto é, correspondente à IC, para os três grupos analisados. Esses dados vão de encontro aos resultados encontrados por Mello ${ }^{16}$, em um grupo de crianças, também de 8 a 10 anos, praticantes de natação. Diferentemente, os autores verificaram que os resultados dos testes de equilíbrio e de organização temporal foram inferiores a todas as idades motoras verificadas nos outros testes. Embora as crianças participantes desse estudo sejam praticantes de uma modalidade esportiva, percebe-se que o desenvolvimento não ocorre igualmente para todos os componentes motores. Particularmente, o equilíbrio, que poderia ser mais influenciado pela prática da natação, conforme demonstrado no estudo de Rosa, Marques, Medina e Gobbi ${ }^{17}$, não pareceu ter sido influenciado pelo exercício.

A não linearidade no desenvolvimento das crianças sugere que a aquisição de habilidades motoras dá-se de forma particular para cada faixa etária e que as restrições para a emergência de padrões complexos de comportamento estão relacionadas com restrições do organismo, do ambiente e da tarefa ${ }^{2}$. Autores ${ }^{1,2}$ defendem que tais restrições influenciam o desenvolvimento motor e mais especificamente, que fatores de crescimento físico podem explicar as mudanças desenvolvimentistas ocorridas no período de avaliação. Essas mudanças parecem ser influenciadas pelas diferenças na estimulação e no encorajamento para explorar seu próprio corpo e o ambiente, podendo privilegiar mais acentuadamente um componente da motricidade em detrimento de outro.

A paralisia cerebral seria um dos tipos de restrição do organismo, e que poderia influenciar no desenvolvimento motor e processo de aprendizagem de habilidades. Em um estudo avaliando o desenvolvimento do equilíbrio em uma criança com paralisia cerebral, Rosa, Marques, MedinaPapst e Gobbi ${ }^{17}$ verificaram mudanças acentuadas, particularmente, no componente equilíbrio, após um programa de intervenção baseado em atividades aquáticas. Assim, embora se considere que a restrição específica influencie no desenvolvimento motor da criança, percebeu-se que a intervenção proporcionou resultados favoráveis sobre um componente particular da motricidade. As autoras concluíram que o programa proporcionou mudanças, muito provavelmente, atribuídas aos aspectos físicos da água e das sessões de atividades propostas.

No caso das crianças com dificuldades de aprendizagem, existe a necessidade de avaliação motora e, com base nos seus resultados, elaborar cuidados à criança referentes ao tipo específico de dificuldade escolar, seja de leitura, compreensão, escrita ou cálculo, assim como às suas necessidades motoras, pois o conhecimento sobre a motricidade das crianças é importante para evitar distúrbios no desenvolvimento ${ }^{18}$. O que vale ressaltar é que pesquisadores têm enfatizado a necessidade de intervenção precoce, assim que detectadas as dificuldades no processo de aprendizagem escolar das crianças ${ }^{19}$.

Ao verificar a relação entre o desenvolvimento físico, motor e rendimento escolar e a análise dos fatores nutricionais em crianças disléxicas e não disléxicas, Rosa Neto, Oliveira, Pires e Luna ${ }^{20}$ verificaram déficits em todas as áreas avaliadas. As idades motoras encontradas foram inferiores ao esperado, com base na idade cronológica das crianças e a escolarização, apresentando corre- 
lação estatisticamente significativa com o rendimento escolar. Os resultados foram explicados, considerando o excesso de tarefas que as crianças apresentam (reforço escolar, atendimento especial multidisciplinar), dificultando a participação dos mesmos em jogos recreativos na escola e no ambiente familiar. Embora se considere tal demanda de atividades, percebe-se a necessidade de uma avaliação do desenvolvimento da criança com o objetivo de elaborar um programa de intervenção específico que permita ao aluno alcançar o grau de desenvolvimento motor e aprendizagem escolar.

Resultados surpreendentes foram encontrados em um estudo, avaliando, também, crianças disléxicas com níveis diferentes de compreensão em leitura. Os autores verificaram uma leitura eficiente das crianças, no entanto, com um nível de compreensão pobre ${ }^{13}$. Além disso, o fato das crianças não estarem estruturadas o suficiente no tempo e no espaço para o aprendizado da leitura e escrita, não possibilita relação positiva e significativa entre a estrutura espaço-temporal e alfabetização ${ }^{21}$. Destaca-se, assim, a importância dos componentes cognitivos, delimitando o desempenho da criança em diferentes áreas da aprendizagem ${ }^{3}$, ou seja, o fator maturacional e sua extrema importância no desenvolvimento da atenção ${ }^{22}$ e no processo de selecionar, processar, reter, armazenar e disponibilizar informações conforme a necessidade. Provavelmente, a falta de atenção, conforme observou Melo, Poeta e Rosa Neto ${ }^{14}$, em um estudo investigando os componentes da motricidade em crianças com hiperatividade e déficit atencional, acarreta carência na concentração para a realização das atividades escolares. Os resultados do estudo desses autores corroboram o presente estudo, no qual foram verificados menores IM nas tarefas de esquema corporal e organização espacial, seguida pela organização temporal. Dessa maneira, o fator atencional e os atrasos motores detectados em crianças com dificuldades de aprendizagem podem estar atrelados ao insucesso nas atividades escolares, tendo em vista a contribuição de tais componentes para o seu desempenho na aprendizagem escolar.

Particularmente, na idade de 8 anos, a noção de corpo e dos sentidos necessita ser dominada para aplicação no processo de aprendizagem da linguagem escrita, sendo o esquema corporal a base para a aprendizagem das noções de espaço e tempo para aplicação nas tarefas escolares ${ }^{23}$. As crianças do presente estudo, em geral, apresentaram menores resultados nos testes de esquema corporal e organização espacial, o que pode estar compro- metendo seu desempenho escolar, acarretando maiores dificuldades. Esses resultados corroboram os achados de Rosa Neto et al. ${ }^{4}$ sobre o desenvolvimento motor de crianças com indicadores de dificuldades de aprendizagem. Os autores revelaram que um número significativo de educandos com dificuldades na aprendizagem escolar apresentaram importante atraso no desenvolvimento motor, bem como condições biopsicossociais adversas. As dificuldades de aprendizagem retratam uma grande diversidade de problemas educacionais e que podem atingir enormes repercussões socioculturais ${ }^{9}$. Corroborando o seu pressuposto, Fiates e Rosa $\mathrm{Neto}^{24}$ confirmaram a relação da aprendizagem escolar e o desenvolvimento psicomotor, concluindo que a observação atenta e precisa dos padrões motores contribui de forma preventiva e reeducativa para amenizar ou excluir fatores impeditivos do potencial de aprendizagem da criança.

Pode-se perceber que há uma preocupação interdisciplinar quanto à detecção precoce das dificuldades escolares, avaliação do nível de desenvolvimento da criança e a necessidade de proposta de intervenção com atividades adequadas, almejando resultados benéficos ao aprendiz e que tendem a reduzir problemas e maiores transtornos futuros nos anos escolares. Além disso, há a necessidade de conhecimento do profissional ligado ao ensino de crianças sobre o processo de desenvolvimento e aprendizagem motora para que este se fundamente na seleção de atividades no processo de intervenção.

\section{CONCLUSÃO}

Diante dos resultados, pode-se concluir que as crianças com dificuldades de aprendizagem apresentaram comprometimento motor no desenvolvimento dos componentes da motricidade, particularmente, aqueles relacionados às noções corporais, espaciais e temporais. Em especial, as crianças mais velhas demonstraram maior diferença entre IC e a IMG, sendo a IC inferior à IM em grande parte dos componentes.

Considerando o maior atraso na área de esquema corporal para todos os grupos, sugere-se a inclusão de tarefas que auxiliem no desenvolvimento dos componentes motores, além das tarefas escolares. Nas habilidades de equilíbrio e motricidade global, apesar das IM alcançadas serem inferiores à IC, indicam uma superioridade desses componentes em relação às outras áreas. Isso pode estar atrelado às próprias experiências advindas das atividades lúdicas e fundamentais tipicamente 
relacionadas à fase infantil que podem contribuir para o desenvolvimento desses componentes em detrimento de outros.

A sugestão de uma análise mais detalhada das tarefas escolares e das dificuldades específicas de aprendizagem escolar, as quais parecem demonstrar relação com os aspectos motores, deve ser foco de maiores estudos, além de um acompanhamento, por meio de avaliação motora, do desenvolvimento motor das crianças ao longo do programa.

\section{REFERÊNCIAS BIBLIOGRÁFICAS}

1. Caetano MJD, Silveira CRA, Gobbi LTB. Desenvolvimento motor de pré-escolares no intervalo de 13 meses. Rev Bras Cineantropom Desempenho Hum 2005;7(2):5-13.

2. Silveira CRA, Gobbi LTB, Caetano MJD, Rossi ACS, Candido RP. Avaliação motora de pré-escolares: relações entre idade motora e idade cronológica. Lecturas: Educación Fisica y Deportes [Periódico on-line]. 2005.

3. Beresford H, Queiroz M, Nogueira AB. Avaliação das relações cognitivas e motoras na aquisição instrucional das habilidades para a aprendizagem da linguagem escrita. Ensaio: aval pol públ Educ 2002;10(37):493-502.

4. Rosa Neto F, Almeida GMF, Caon G, Ribeiro J, Caram JA, Piucco EC. Desenvolvimento motor de crianças com indicadores de dificuldades na aprendizagem escolar. Rev Bras Cien Mov 2007;15(1):45-51.

5. Rosa Neto F. Manual de avaliação motora. Porto Alegre: Artmed, 2002.

6. Willrich A, Azevedo CCF, Fernandes JO. Desenvolvimento motor na infância: influência dos fatores de risco e programas de intervenção. Rev Neurocienc 2009;17(1):51-56.

7. Medeiros PC, Loureiro SR, Linhares MBM, Maturano EM. A auto-eficácia e os aspectos comportamentais de crianças com dificuldade de aprendizagem. Psicol Refl Crít 2000; 13(3):327-336.

8. Okano CB, Loureiro SR, Linhares MBM, Maturano EM. Crianças com Dificuldades Escolares Atendidas em Programa de Suporte Psicopedagógico na Escola: Avaliação do Autoconceito. Psicol Refl Crít 2004;17(1):121-128.

9. Moreira NR, Fonseca V, Diniz A. Proficiência motora em crianças normais e com dificuldade de aprendizagem: estudo comparativo e correlacional com base no teste de proficiência motora de Bruininks-Oseretsky. Rev Educ Física 2000; 11(1): 11-26.

10. Nascimento R, Madureira VSF, Agne JE. Avaliação do desenvolvimento neuropsicomotor de crianças em Centros de Educação Infantil em Concórdia. Rev Neurocienc 2008;16(4):284-291.

11. Gomes CCP, Pavão S. M. O. Dificuldades de aprendizagem. Educação 2001; Santa Cruz.

12. Baltazar MO. Tratamento das dificuldades de aprendizagem de leitura e escrita à luz da psicopedagogia construtivista. Reflexão e Ação 2001;09(1):37-46.

13. Nation K, Clarke P, Snowling MJ. General cognitive ability in children with reading comprehension difficulties. Br J Educ Psychol 2002;72(4):549-560.

14. Mello LM, Poeta LS, Rosa Neto F. Aptidão motora em escolares com hiperatividade (TDAH) do ensino fundamental de Florianópolis, SC (Resumo). Motriz 2003;9(1):S109-200.

15. Crippa LR, Souza JM. Estudo de parâmetros motores em pré-escolares: organização espacial, organização temporal e esquema corporal. In: Krebs RJ, organizador. II Encontro latino-americano para estudos da criança: desenvolvimento infantil. Florianópolis: UDESC; 2002. p. 60.

16. Mello RRF. O perfil motor de alunos atletas de 8 a 10 anos que praticam regularmente natação. [Monografia de Graduação - Centro de Educação Física, Fisioterapia e Desportos]. Florianópolis (SC): Universidade Estadual de Santa Catarina; 1997.

17. Rosa GKB, Marques I, Medina-Papst J, Gobbi LTB. Desenvolvimento motor de criança com paralisia cerebral: avaliação e intervenção. Rev bras educ fís Esp 2008;14(2):163-176.

18. Leite L, Rosa Neto F, Franceschi CR. Perfil motor em praticantes precoces de futebol de salão. [Monografia de Graduação - Centro de Educação Física, Fisioterapia e Desportos]. Florianópolis (SC): Universidade Estadual de Santa Catarina; 1997.

19. Lamb SJ, Bibby PA, Wood DJ, Leyden G. An intervention programme for children with moderate learning difficulties. Br J Educ Psychol 1998;68(4):493-504.

20. Rosa Neto F, Oliveira AJ, Pires MMS, Luna JLS. Perfil biopsicossocial de crianças disléxicas. Temas em Desenvolvimento 2000;9(51):21-24.

21. Alvim MPB, Borges OP. Análise da relação da estrutura espaço-temporal e alfabetização em crianças de 6 a 7 anos. (Resumo). Rev Port Ciên Desp 2004;04(2):164.

22. Ladewig I. A importância da atenção na aprendizagem de habilidades motoras. Rev paul educ física 2000;14(supl.3):62-71.

23. Medina J, Rosa GKB, Marques I. Desenvolvimento da organização temporal de crianças com dificuldades de aprendizagem. Rev educ Fís/UEM 2006; 17(1): 107-116.

24. Fiates MP, Rosa Neto F. Estudo da relação entre o desenvolvimento psicomotor e as dificuldades na aprendizagem de um grupo de crianças de 4 a 7 anos. 2001. [Monografia de Especialização em Educação Especial - Centro de Educação Física, Fisioterapia e Desportos]. Florianópolis (SC): Universidade Estadual de Santa Catarina; 2001.

\section{Endereço para correspondência}

Josiane Medina Papst

Rua Fernando de Noronha, 197, apt. 202, Centro CEP: 86020-300 - Londrina. PR. Brasil

E-mail: josi_medina@hotmail.com 